\title{
Le Pays-de-Retz au début du Mésolithique : le site des Vingt-Deux Boisselées-1 (Saint-Père-en-Retz, Loire-Atlantique)
}

The Pays-de-Retz and the Early Mesolithic: The Site of "les Vingt-Deux

Boisselées1" (Saint-Père-en-Retz, Loire-Atlantique)

Sylvène Michel

\section{OpenEdition Journals}

Édition électronique

URL : http://journals.openedition.org/rao/1439

DOI : 10.4000/rao.1439

ISBN : 978-2-7535-1846-9

ISSN : $1775-3732$

Éditeur

Presses universitaires de Rennes

Édition imprimée

Date de publication : 31 décembre 2011

Pagination : 19-36

ISBN : 978-2-7535-1844-5

ISSN : 0767-709X

Référence électronique

Sylvène Michel, « Le Pays-de-Retz au début du Mésolithique : le site des Vingt-Deux Boisselées-1

(Saint-Père-en-Retz, Loire-Atlantique) », Revue archéologique de l'Ouest [En ligne], 28 | 2011, mis en ligne le 30 mars 2014, consulté le 03 décembre 2020. URL : http://journals.openedition.org/rao/1439 ; DOI : https://doi.org/10.4000/rao.1439 


\title{
Le Pays-de-Retz au début du Mésolithique : le site des Vingt-Deux Boisselées-1 (Saint-Père-en-Retz, Loire-Atlantique)
}

\author{
The Pays-de-Retz and the Early Mesolithic: \\ The Site of "les Vingt-Deux Boisselées1" (Saint-Père-en-Retz, Loire-Atlantique)
}

\author{
Sylvène MicheL*
}

\begin{abstract}
Résumé : De récents sondages permettent de compléter la caractérisation de l'occupation mésolithique des Vingt-Deux Boisselées-1 (Saint-Père-enRetz, Loire-Atlantique), site auparavant prospecté et servant de référence régionale depuis une douzaine d'années. La série, homogène d'un point de vue chrono-culturel, est principalement réalisée en silex blond-jaune. Les supports sont obtenus suivant des modalités simples, nécessitant un faible investissement techno-économique. Ils sont pour certains peu transformés et constituent alors des outils du fonds commun, et pour d'autres finement retouchés comme en témoignent les armatures. L'analyse typologique des microlithes issus du sondage 1 dévoile des résultats différents de ceux obtenus suite aux prospections (plus de triangles que de pointes à base naturelle et non l'inverse), ce qui ouvre peut-être de nouvelles pistes d'étude en termes de classification chrono-culturelle et incite à interpréter avec prudence les scénarios construits à partir de simples ramassages.
\end{abstract}

\begin{abstract}
Recent excavations allow to complete the characterization of the Mesolithic site of the Vingt-Deux Boisselées-1, serving as regional reference since a dozen years. The lithic industry, chrono-culturally homogeneous, is mainly realized in blond-yellow flint. Supports are obtained from simple modalities, requiring a weak techno-economic investment. They are for some little transformed and make up then common tools, and for of the other ones finely retouched as show of it the arrowheads. The typological analysis of arrowheads found during the excavation reveals different results from those obtained further to the surveys (more triangles than points with natural base and not the opposite), what opens maybe new avenues of research in terms of chrono-cultural classification.
\end{abstract}

Mots clés : industrie lithique, premiers groupes mésolithiques, ouest de la France, étude techno-économique, caractérisation chrono-culturelle.

Keywords : lithic industry, Early Mesolithic, Western France, techno-economic study, chrono-cultural characterization.

La basse vallée de la Loire bénéficie depuis la fin des années 1970 de nombreuses prospections pédestres alimentant le corpus des sites attribuables aux premiers groupes mésolithiques (seconde moitié du X $\mathrm{X}^{\mathrm{e}}$ millénaire et IX $\mathrm{X}^{\mathrm{e}}$ millénaire avant notre ère). Quelques fouilles programmées ont par la suite été menées pour essayer de pallier la fréquente hétérogénéité de ces ensembles archéologiques de surface et tenter de mieux appréhender la nature de l'ha- bitat, mais aussi l'organisation techno-économique de ces chasseurs-collecteurs et leur géographie chrono-culturelle. Malgré les efforts déployés, plusieurs opérations n'ont pas pu répondre à ces problématiques : une combinaison de processus bio-chimiques et mécaniques n'a souvent pas permis la conservation des sites sondés, sites mêlant alors dans une seule et même couche toute une succession d'occupations préhistoriques (Gouraud, 1990; Marchand et

*INRAP Grand-Ouest/UMR 6566 CReAAH - 6 place de la Mairie, 35250-Saint Aubin-d'Aubigné. (sylvene.michel@hotmail.fr) 
al., 1999). Dans l'actuelle Loire-Atlantique, seules deux campagnes de fouilles ont été couronnées de succès, en mettant au jour des ensembles homogènes. La première concerne l'Organais à Sainte-Reine-de-Bretagne, site exploré sur $17 \mathrm{~m}^{2}$ (Gallais et al., 1985; Le Goff, 2003). En avril 2010, le matériel lithique a fait l'objet d'une nouvelle étude partielle, quatre cinquièmes du matériel demeurant alors introuvables (Michel, 2011 p. 228-240). Le complément d'étude devrait pouvoir être mené sous peu puisque l'ensemble du matériel a tout récemment été redécouvert. Dans cet article, nous allons donc nous concentrer sur le second cas, soit les Vingt-Deux Boisselées-1, site sondé en 2009 dans le Pays-de-Retz.

\section{Des PRospeCtions AUX SONDAGES : PRÉSENTATION GÉNÉRALE DU SITE DES ViNGT-DEUX BoISSELÉS-1}

Entre 1997 et 2000, des ramassages de surface sur moins d'1 $\mathrm{km}^{2}$ ont permis à $\mathrm{M}$. Tessier de mettre au jour six concentrations de vestiges mésolithiques sur la commune de Saint-Père-en-Retz (Loire-Atlantique, fig. 1). La distance qui sépare ces découvertes (de l'ordre de 200 à $800 \mathrm{~m}$ ) ne justifie pas l'emploi du terme locus proposé à l'origine par le prospecteur. Cet article va se concentrer sur l'habitat pour lequel le matériel s'est avéré le plus homogène et le plus abondant : le site des Vingt-Deux Boisselées1, anciennement nommé locus 1. Plus de 3000 pièces lithiques attribuables au début du Mésolithique ont été récoltées sur une même parcelle dans un périmètre restreint à une soixantaine de mètres (fig. 2). Cet ensemble de surface a fait l'objet d'une étude amorcée par M. Tessier et complétée par G. Le Goff dans le cadre d'un travail universitaire (Tessier, 1998; Le Goff, 2002).

Grâce à la cohérence chrono-culturelle du mobilier découvert en prospection, les Vingt-Deux Boisselées-1 ont immédiatement servi de site référent pour le Mésolithique régional et ont ainsi été sollicités par de nombreux travaux de synthèse à l'échelle de l'ouest de la France. Pour documenter au mieux cette occupation et évaluer son degré de conservation, une série de trois sondages a été entreprise à l'été $2009^{1}$ (Michel, 2009). Seul le sondage de $4 \mathrm{~m}^{2}$ réalisé dans la partie méridionale de la parcelle, où les prospections avaient été fructueuses, a fourni du matériel archéologique conséquent (fig. 2, sondage 1). Dans les deux autres - respectivement implantés dans un espace

1. M. Tessier avait déjà réalisé de petits sondages manuels $\left(0,25 \mathrm{~m}^{2}\right)$ très vite avortés; l'exiguïté de ces fenêtres explique le fait que l'archéologue n'ait pas détecté de vestiges dans le niveau infra-labour, contrairement à l'opération de 2009. tourbeux n'ayant pas été labouré et dans une zone basse où les dépôts de pente accumulés auraient pu préserver les couches archéologiques - les indices archéologiques se sont en effet montrés des plus discrets.

Dans le sondage 1, sous le labour, on ne distingue qu'une couche de sables et graviers pliocènes, sans aucune unité stratigraphique distincte. Des passes arbitraires de $5 \mathrm{~cm}$ d'épaisseur ont donc été réalisées. La fouille et l'enregistrement des données ont pris pour échelle le quart de mètre carré et les sédiments ont systématiquement été tamisés à l'eau. La couche pliocène est moins épaisse en ce lieu qu'aux alentours (Ters et al., 1978) puisque le socle rocheux est apparu vers $60 \mathrm{~cm} \mathrm{de}$ profondeur. La pente du terrain est axée Sud-Ouest/NordEst, avec un dénivelé de 2,8\% au niveau des Vingt-Deux Boisselées-1, soit 5,8 m entre le haut et le bas de la parcelle. Le site prend place sur le flanc nord du relief créé par une faille tertiaire axée Ouest-Est conditionnant la topographie globale du Pays de Retz. Elle sépare, au Sud, un plateau allant jusqu’à $60 \mathrm{~m}$ d'altitude, et au Nord, une vaste zone déprimée (ibid.). Celle-ci accueille la vallée du Boivre, fleuve qui naît à $400 \mathrm{~m}$ seulement du site étudié.

D'après le sondage 1 , la dégradation du site semble importante : plus des deux tiers des vestiges ont été découverts dans les terres récemment labourées (tabl. 1). Sous le labour, les vestiges ont été découverts sur $30 \mathrm{~cm}$ de haut mais, au-delà des $15 \mathrm{~cm}$ supérieurs, leur occurrence est des plus discrètes (tabl. 1, N4-N6). Compte tenu de la nature sablo-graveleuse du sédiment, il est possible que plus une seule pièce archéologique ne soit dans sa position d'origine. On notera néanmoins que la probable migration verticale des vestiges est de faible ampleur par rapport à d'autres sites mésolithiques découverts en contexte sableux (Barton, 1987; Crombé, 1993; Vermeersch, 1976, 1995).

Certes cette opération n'a livré aucun sol d'habitat ou structure, pas plus que des renseignements quant à l'organisation spatiale de ce site, mais les informations qu'apportent les vestiges mobiliers sont néanmoins conséquentes. Comme l'avaient fortement suggéré les prospections, et si l'on s'en réfère aux armatures qui en l'absence de vestiges organiques datables sont les seuls éléments permettant d'évaluer le degré de cohérence de l'ensemble, l'industrie lithique est clairement homogène. En plus du mobilier mésolithique, le sondage a livré quelques petits tessons, assez hétérogènes et tous localisés dans le labour. En conséquence, le site des Vingt-Deux Boisselées-1 offre la possibilité d'analyser une série archéologique mono-culturelle (occupation ou retours successifs d'individus appartenant à la même tradition culturelle). 


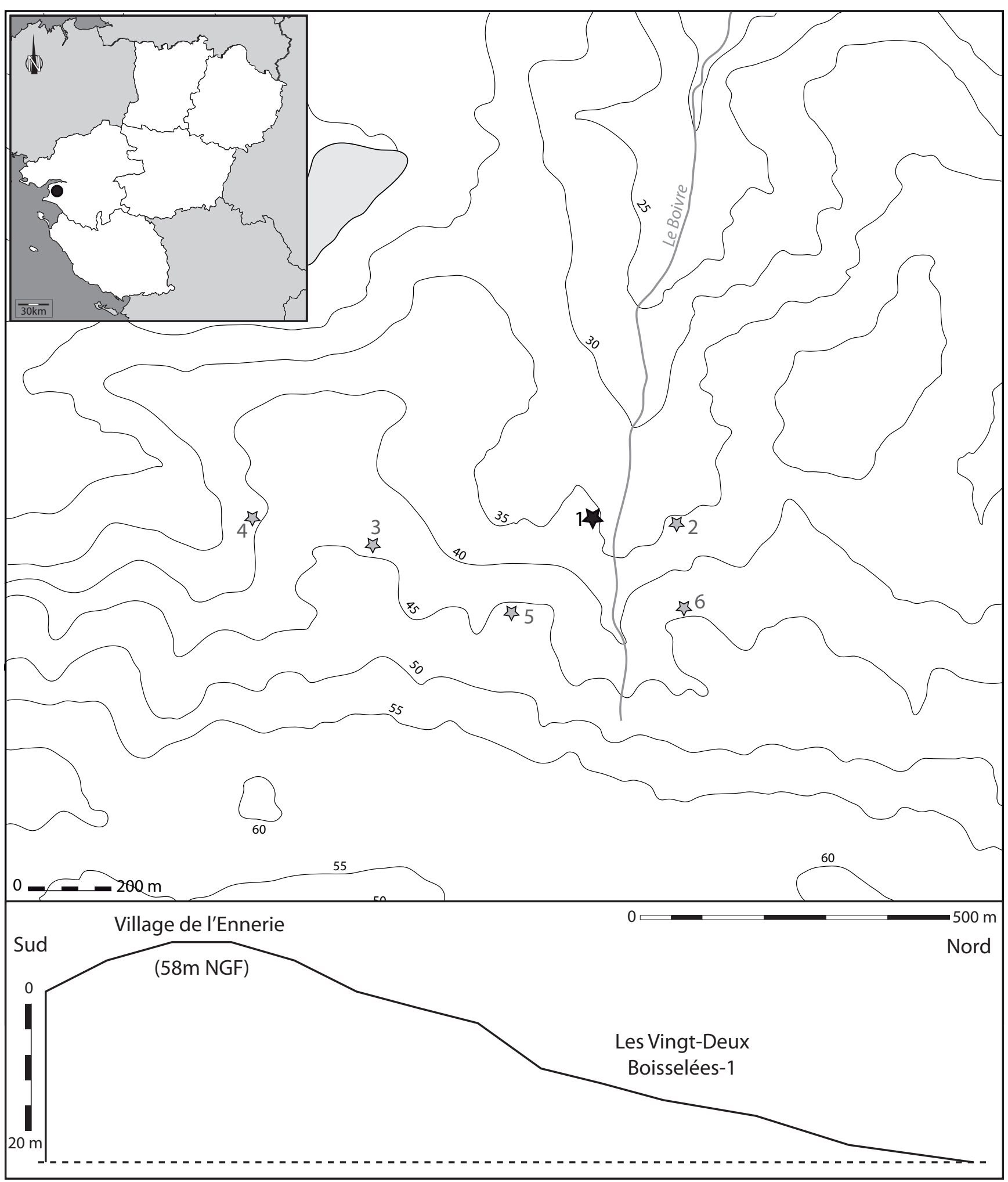

Figure 1 : Localisation des six sites des Vingt-Deux Boisselées dans la vallée du Boivre. Le site qui fait l'objet de cet article est représenté par l'étoile noire. Sur le profil, en bas de la figure, les altitudes ont été multipliées par 10. Fonds de carte Pays-de-la-Loire [histgeo.ac-aixmarseille.fr]. Courbes de niveau d'après la carte IGN au 1/25000.

Figure 1: Location of the six sites des Vingt-Deux Boisselées in the valley of Boivre. The site which is the object of this article is represented by the black star. On the profile, at the bottom of the figure, the heights are multiplied by ten. 


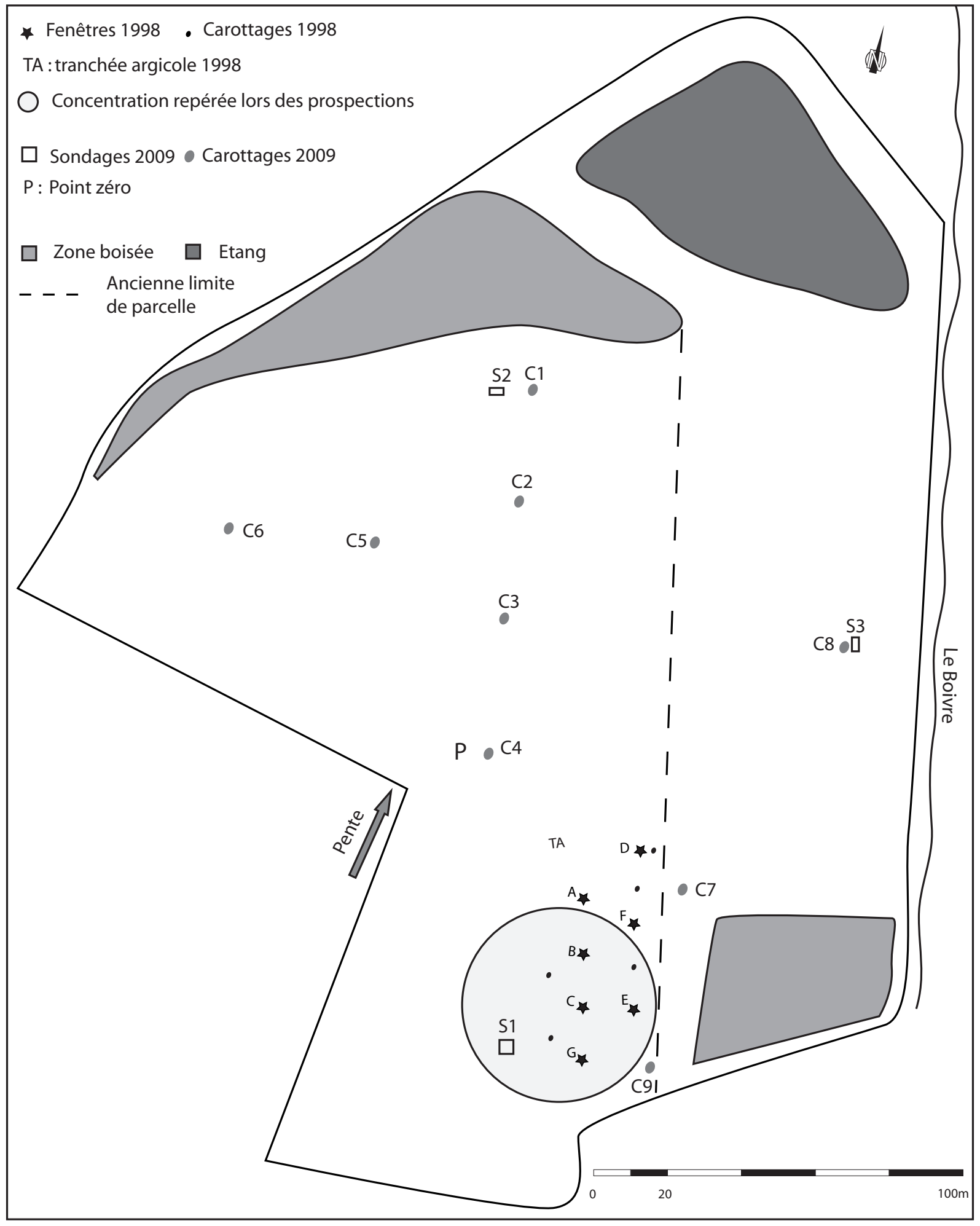

Figure 2 : Plan des opérations archéologiques menées au site des Vingt-Deux Boisselées-1 entre 1997 et 2009. La dimension des sondages et carottages a été augmentée pour faciliter la lecture.

Figure 2: Plan of the archaeological operations led to the site des Vingt-Deux Boisselées-1 between 1997 and 2009. The dimension of the excavations was increased to facilitate the reading. 


\begin{tabular}{|c|c|c|c|}
\hline Passes archéologiques & N & $\%$ & $\%$ hors labour \\
\hline Labour & 1276 & 67,6 & - \\
\hline N1 & 269 & 14,2 & 44,0 \\
\hline N2 & 194 & 10,3 & 31,7 \\
\hline N3 & 105 & 5,6 & 17,2 \\
\hline N4 & 22 & 1,2 & 3,6 \\
\hline N5 & 20 & 1,1 & 3,3 \\
\hline N6 & 2 & 0,1 & 0,3 \\
\hline Total & 1888 & 100 & 100 \\
\hline
\end{tabular}

Tableau 1 : Répartition verticale du matériel lithique au sein du sondage 1, exprimée en nombre et en pourcentage (en incluant et excluant les terres labourées).

Tableau 1: Vertical distribution of the lithic material.

\section{Caractères de la production lithiQue}

\section{Les matières premières débitées}

L'assez faible taux de pièces patinées ou détériorées par le $\mathrm{feu}^{2}$ permet de déterminer les matières premières dans près de neuf cas sur dix. Un peu plus de $89 \%$ des vestiges pour lesquels la matière première a pu être caractérisée sont constitués d'un silex blond-jaune, plus ou moins translucide et de trame assez uniforme ${ }^{3}$. Cette matière, d'aspect lisse, est le plus souvent homogène; cependant, certains volumes abandonnés avant d'avoir fait l'objet d'une réelle exploitation montrent que les fissures et les inclusions mal silicifiées ne sont pas rares, ce qui entraine parfois l'explosion des nodules. Ces derniers se présentent sous la forme de galets dont le transport, ponctué de nombreux chocs, explique les fissurations précitées; en effet, les vestiges comportant encore la gangue corticale témoignent de l'aspect roulé du cortex, également caractérisé par sa faible épaisseur. On soulignera la petitesse des galets à peine ébauchés - jusqu’à une quarantaine de millimètres de diamètre minimum - caractéristique qui se retrouve dans les galets de silex blond-jaune encore accessibles au sud de l'embouchure de la Loire, et plus exactement à une vingtaine de kilomètres, à Préfailles au niveau de la Pointe Saint-Gildas. Des ramassages systématiques de galets en ce lieu ont permis à $\mathrm{M}$. Tessier de montrer que leur nombre décroît en fonction de leur allongement, qui peut atteindre $90 \mathrm{~mm}$; au-delà des $60 \mathrm{~mm}$, les quantités s'amenuisent tout de même nettement, ce qui est peut-être à mettre en lien avec une collecte sélective et répétée au cours

2. Les pièces brûlées représentent $18,3 \%$ de la série.

3. Ces observations reposent sur un examen à l'œil nu et à la loupe binoculaire à faible grossissement.

de la Préhistoire en faveur des modules les plus importants (Tessier cité par Marchand, 1997, p. 54-55). Dans le cas présent on insistera sur le fait que les plus grands enlèvements décomptés ne mesurent qu'une quarantaine de millimètres de long.

Les autres matières siliceuses ne jouent qu'un rôle secondaire dans le débitage. Ceci n'est pas un trait propre à cette série et il s'observe de manière récurrente dans les collections mésolithiques régionales, dont celle de la Fillauderie pour ne citer que l'exemple le plus proche (Saint-Père-en-Retz, LoireAtlantique; Marchand et al., 1999). Dans le sondage 1 des Vingt-Deux Boisselées-1, on trouve d'anecdotiques esquilles et fragments de lamelles en jaspe et calcédoine, alors que le silex des Moutiers et les grès-quartzites représentent respectivement $6,5 \%$ et $4 \%$ du matériel. La provenance du silex des Moutiers est clairement identifiée, son gîte se situant dans la baie de Bourgneuf à un peu moins d'une vingtaine de kilomètres. L'approvisionnement en grès-quartzite pose plus de questions. L'affleurement de Montbert, à un peu moins d'une cinquantaine de kilomètres de Saint-Père-enRetz, est bien connu par les préhistoriens depuis le $\mathrm{XIX}^{\mathrm{e}}$ siècle (Gouraud et al., 1990), mais il ne faut pas oublier que de nombreuses dalles de grès-quarztites à grains fins sont dispersées en Sud-Loire/Nord-Vendée (Arrivé et al., en préparation). Des prospections à visée pétrographique seraient nécessaires pour vérifier l'éventuelle corrélation entre les pièces archéologiques et les spécimens issus des différents gisements régionaux. Soulignons tout de même pour le site étudié la forte probabilité d'un approvisionnement plus local qu'une récolte sur le gîte montbertain, et par exemple à une quinzaine de kilomètres de Saint-Père-en-Retz aux Moulins des Penauds ou à la Bitaudière, au sommet de sables blancs yprésiens identiques à ceux de Montbert (Ters et al., 1979, p. 11-12).

Ces deux dernières matières sont attestées par des enlèvements bruts ou parfois retouchés. Notons au passage que les quelques armatures et pièces du fonds commun fabriquées dans ces roches ne correspondent pas à des types spécifiques et différents de ceux réalisés en silex blond. Les nucléus sont quant à eux rares dans le cas du silex des Moutiers et même absents du sondage 1 en ce qui concerne le grès-quartzite. On ne peut cependant conclure à l'introduction de cette dernière matière sur le site uniquement sous forme de supports bruts ou retouchés puisque la prospection a livré plusieurs nucléus constitués de grès-quartzite. Cela nous amène à évoquer les modalités de transport du silex blond. Les proportions de pièces semi-corticales et corticales (représentant respectivement $9,5 \%$ et $5,3 \%$ des produits bruts de cette matière) ainsi que le nombre important de galets abandonnés aux premiers coups portés (tabl. 2) plaideraient plutôt en faveur d'un import de galets bruts ou juste décalottés, 


\begin{tabular}{|c|c|c|c|}
\hline Catégorie & Fragmentation & $\mathrm{N}$ & $\%$ \\
\hline \multirow{2}{*}{ Éclat } & entier & 199 & 13,7 \\
\hline & fragment & 307 & 21,1 \\
\hline \multirow{2}{*}{ Lame } & entier & 3 & 0,2 \\
\hline & fragment & 49 & 3,4 \\
\hline \multirow{2}{*}{ Lamelle } & entier & 17 & 1,2 \\
\hline & fragment & 602 & 41,3 \\
\hline \multicolumn{2}{|c|}{ Nucleus } & 16 & 1,1 \\
\hline \multicolumn{2}{|c|}{ Bloc mis en forme/Casson } & 35 & 2,4 \\
\hline \multicolumn{2}{|c|}{ Crête, néo-crête } & 7 & 0,5 \\
\hline \multicolumn{2}{|c|}{ Tablette } & 9 & 0,6 \\
\hline \multicolumn{2}{|c|}{ Microburin } & 61 & 4,2 \\
\hline \multicolumn{2}{|c|}{ Armature } & 62 & 4,3 \\
\hline \multicolumn{2}{|c|}{ Outil aménagé } & 31 & 2,1 \\
\hline \multicolumn{2}{|c|}{ Chute de burin } & 2 & 0,1 \\
\hline \multicolumn{2}{|c|}{ Pièce à fil ébréché } & 57 & 3,9 \\
\hline \multicolumn{2}{|c|}{ Sous-total } & 1457 & 100 \\
\hline \multicolumn{2}{|c|}{ Esquille } & 196 & \\
\hline \multicolumn{2}{|c|}{ Débris } & 235 & \\
\hline \multicolumn{2}{|c|}{ TOTAL } & 1888 & \\
\hline
\end{tabular}

Tableau 2 : Composition globale de la série lithique récoltée dans le sondage 1 .

Tableau 2: Global composition of the lithic industry.

mais pas décortiqués ni testés sur leur lieu d'acquisition. En témoigne la relative fréquence d'accidents prématurés dus à des défauts de matière première; cela est peut-être lié au caractère facilement renouvelable de l'approvisionnement.

\section{Décryptage des séquences de débitage}

Compte tenu de la composition pétrographique de la collection, la description globale des séquences de débitage va être restreinte aux vestiges taillés dans le silex blond ligérien.

\section{Ouverture et mise en forme des galets}

L'ouverture des galets n'appelle visiblement pas aux VingtDeux Boisselées-1 la percussion bipolaire sur enclume, les vestiges témoignant de cette méthode-technique y étant quasiment inexistants. Cette observation s'accorde avec une synthèse régionale soulignant le caractère très ponctuel de ce type de percussion sur l'ensemble des sites mésolithiques de l'ouest de la France (Guyodo et Marchand, 2005). Les stigmates observables en partie proximale des enlèvements détachés au cours des premières séquences opératoires sont marqués - talon épais avec trace d'impact, fissurations, ridules et étoilures - et typiques de l'utilisation d'un percuteur de pierre dure.
Certains de ces éclats (éclats stricto sensu ou laminaires) permettent de dégager simplement des nervures guides tout en respectant le caractère nécessairement économe en matière de la mise en forme. Celle-ci s'avère en effet rudimentaire, du fait des caractéristiques morphologiques et morphométriques précédemment décrites à propos des galets, et elle correspond certainement en grande partie à un décorticage sommaire. En l'absence de remontages, il faut souligner que les phases de débitage comprises entre l'ouverture des galets et leur exploitation lamellaire ne sont pas encore précisément documentées. Se cache peut-être ici une séquence d'extraction de quelques éclats assez peu épais destinés à la fabrication d'outils du fonds commun, production qui s'insèrerait alors dans un processus global visant au final à l'obtention de lamelles. Il convient à ce stade de la réflexion de signaler qu'un seul nucléus, à table large semitournante exploitée par deux plans de frappe, témoigne en fin de débitage de la production intentionnelle d'éclats. Les séquences de production laminaire ne sont quant à elles pas représentées par les nucléus; les enlèvements lamino-lamellaires larges de plus de $9 \mathrm{~mm}$ sont également rares parmi les produits bruts (tabl. 2) et retouchés.

\section{Modalités de production lamellaire}

La production lamellaire joue un rôle important sur le site des Vingt-Deux Boisselées- 1 comme en témoignent à la fois le taux de lamelles de moins de $10 \mathrm{~mm}$ de large $(52,6 \%$ des enlèvements bruts et un peu plus des trois quarts des supports retouchés) et les nucléus qui, à une exception près, portent les stigmates d'une exploitation lamellaire.

La transformation d'éclats en supports de production lamellaire est ponctuelle dans la collection. Ce cas de figure se résume à trois spécimens en silex blond et un autre en silex des Moutiers (fig. 3, n 3). D'épais éclats encore partiellement corticaux, provenant de la mise en forme des galets ou dans un cas certainement de leur simple fracturation, ont été exploités à ces fins. Les débitages sont alors variés, avec pour premier exemple un fragment répondant au schéma $\mathrm{du}$ " nucléus de type rabot ». Sur une autre pièce, le débitage lamellaire s'effectue en partie proximale de l'éclat, aux dépens de la face inférieure, suivant une exploitation semitournante, unipolaire et orthogonale à l'axe de l'éclat support. On observe également un cas d'exploitation sur la face inférieure, parallèlement au sens de débitage du support; ce n'est pas la longueur potentielle des lamelles qui est ainsi recherchée puisque l'artisan ne s'enquiert pas de supprimer le bulbe marqué de l'éclat-support. Aucun aménagement ne vient d'ailleurs faciliter le débitage de ces trois pièces, ce qui explique en partie l'apparition rapide d'accidents. Il est clair que ces différents nucléus résultent clairement d'un débitage d'appoint peu productif. 


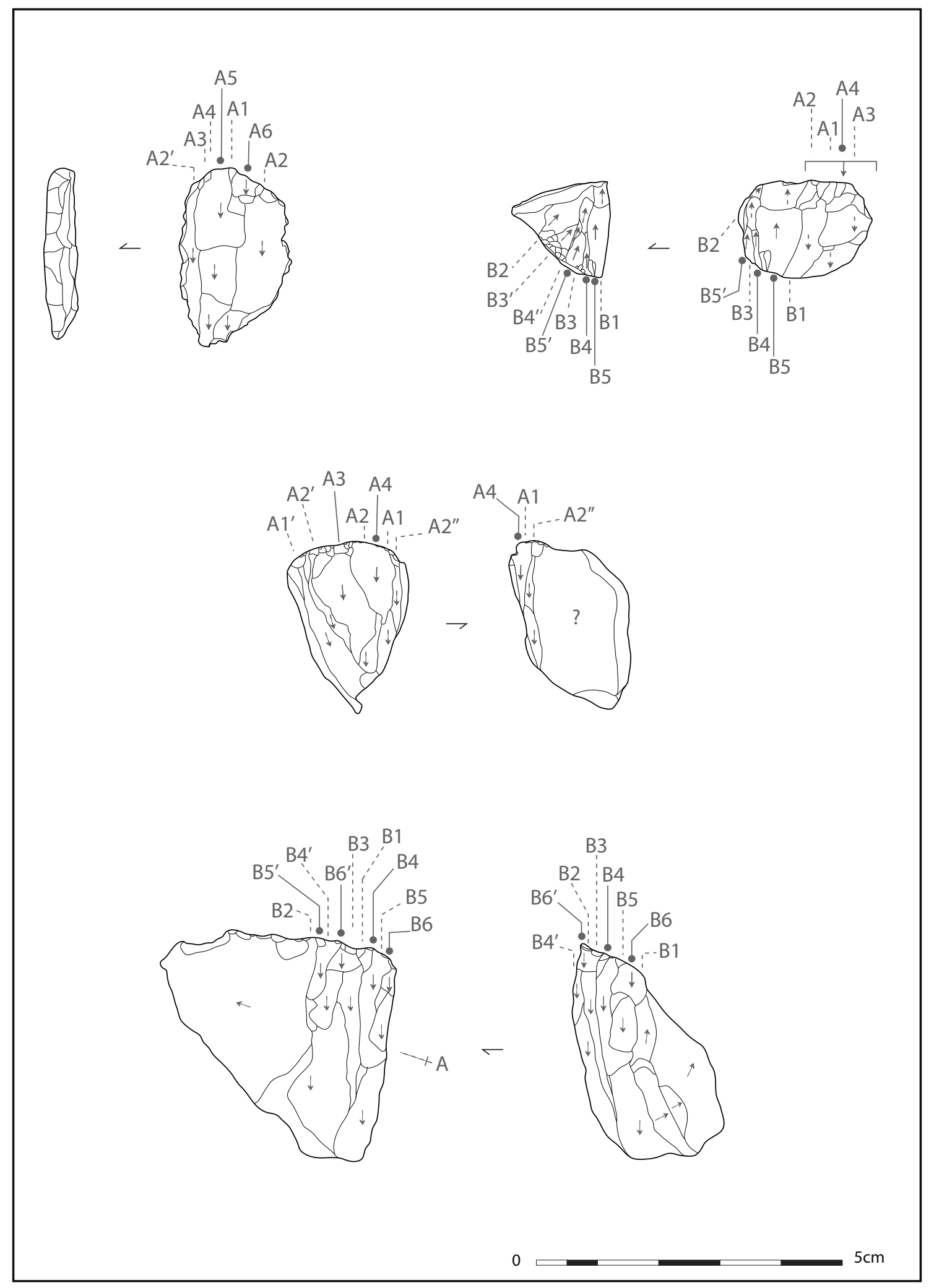

Figure 3 : Nucléus (celui du bas est un nucléus sur éclat, la face inférieure correspondant au flanc gauche).

Figure 3: Cores from the excavations. 
Dans le cas où le volume débité correspond à l'origine à un galet et non à un éclat (fig. 4 et 5), les tailleurs n'ont pas cherché à implanter la table dans une partie étroite du nodule ou à en conserver l'axe longitudinal. Le débitage n'occupe très souvent qu'une table, avec une exploitation semi-tournante qui ne s'effectue pas strictement de gauche à droite ou vice versa mais de manière plus discontinue comme en témoignent les schémas diacritiques et les codes opératoires enregistrés. Deux plans de frappe - essentiellement opposés - interviennent souvent dans le débitage (54,5\%), mais néanmoins les séquences de production lamellaire peuvent être qualifiées d'unipolaires (fig. 4). En effet, bien souvent la surface opposée au plan de frappe principal est percutée dans le but de réaménager la carène. Dans quelques autres cas, le second plan de frappe est également à l'origine du détachement de lamelles. Le débitage se décompose alors en deux séquences indépendantes et successives, respectivement obtenues grâce à l'un puis à l'autre des plans de percussion. Un des nucléus en silex des Moutiers s'inscrit dans ce cas de figure (fig. $5, \mathrm{n}^{\circ} 1$ ) : la production gagne toute la périphérie du volume exploitée depuis deux pôles de percussion désaxés l'un par rapport à l'autre, ce qui confère un aspect spiralé à cette pièce.

Sur l'ensemble de la série, les plans de frappe, lisses, ne sont que sommairement préparés. La seule préparation de la zone à percuter, en l'occurrence l'abrasion des corniches, n’apparaît que ponctuellement sur les nucléus abandonnés. En revanche, au sein du débitage brut, une lamelle sur deux en porte les stigmates. Les talons associés peuvent ponctuellement être filiformes mais, plus généralement, ils sont lisses et larges d'1 ou $2 \mathrm{~mm}$. L'angle talon/axe de débitage approche l'orthogonale ou $80^{\circ}$. En suivant les critères avancés par J. Pelegrin (Pelegrin, 2000), ces différentes observations orientent le raisonnement vers l'utilisation d'un percuteur de pierre en percussion directe; la dureté de l'outil employé reste à définir avec plus de précision, ce qui nécessiterait une série d'expérimentation sur des galets récoltés à l'embouchure de la Loire.

Pour caractériser les lamelles produites par ces débitages, on peut tout d'abord retenir qu'en termes de largeur la production suit une courbe unimodale, avec pour modules préférentiels les valeurs comprises entre 6 et $9 \mathrm{~mm}$. Les tailleurs visent l'obtention de produits fins, globalement de $2 \mathrm{~mm}$ même si les pièces d'1 $\mathrm{mm}$ d'épaisseur ne sont pas en reste. La forte fragmentation des lamelles diminue nos chances d'appréhender la longueur de ces produits, mais il est clair que l'exploitation n'est pas réduite à des lamelle stricto sensu et que des éclats lamellaires se mêlent à la production. Les lamelles largo sensu, souvent torses, possèdent majoritairement deux pans mais la présence d'un pan supplémentaire n'est pas rare. Elles présentent globalement des nervures et/ ou des bords subparallèles légèrement sinueux, sans que les tailleurs n'aient pour autant recherché des produits réguliers.

\section{Entretien et abandon des volumes}

Les enlèvements participant au réaménagement du plan de frappe sont assez peu nombreux (tabl. 2). De minces éclats, tirés depuis la table lamellaire ou un de ses flancs, sont alors privilégiés. On note que, plus globalement, les réaménagements du volume faisant appel à des modalités spécifiques s'avèrent plutôt rares, comme en témoigne également le nombre réduit de néo-crêtes. La correction des convexités survient alors à la suite d'un aménagement partiel d'un pan. Ces deux types d'opérations semblent circonscrits aux séquences initiales et/ou intermédiaires de production lamellaire, les nucléus n'en faisant plus état. La série n'atteste pas non plus de réagencements importants du volume ou d'exploitations par fréquents retournements du bloc, ce qu'indiquent les stigmates des nucléus mais aussi la large prédominance des enlèvements évoquant un débitage unipolaire. En définitive, l'entretien ne nécessite souvent que l'intervention de simples enlèvements, permettant à eux seuls de supprimer les réfléchissements ou de retrouver des carène et cintre satisfaisants. Le débitage semi-tournant permet pour sa part le maintien de ce dernier. Les dos, quant à eux, entrent très peu en jeu dans la logique d'aménagement et sont majoritairement corticaux.

L’abandon du nucléus est généralement causé par la présence concomitante de réfléchissements et d'un aplatissement marqué des convexités. Certes dans certains cas de figure, l'arrêt du débitage est dû à l'exhaustion du volume exploitable (fig. $3, \mathrm{n}^{\circ} 2$ ), mais il convient de souligner que la petitesse initiale qui caractérise les galets taillés n’a pas induit des stratégies de débitage visant à leur exploitation maximale, pas plus qu'un développement important du débitage sur éclat qui s'avère une solution adéquate en cas de gestion plus exhaustive de la matière.

\section{L’outillage retouché}

\section{Un outillage orienté vers des activités cynégétiques?}

Cette apostrophe introduisant l'étude de l'outillage ne vise qu’à rappeler la difficile évaluation de la nature des activités menées par les premiers groupes mésolithiques sur les sites armoricains ou plus généralement de l'ouest de la France. En effet, pour amorcer ce type de réflexion, il faut se contenter de la seule industrie lithique (les ossements n'étant pas conservés), sans omettre de préciser de surcroît l'insuccès très fréquent des analyses tracéologiques avortées par des altérations post-dépositionnelles. Or, force est de constater que la proportion armatures/outils du fonds commun ne peut à elle seule permettre de proposer une caractérisation 


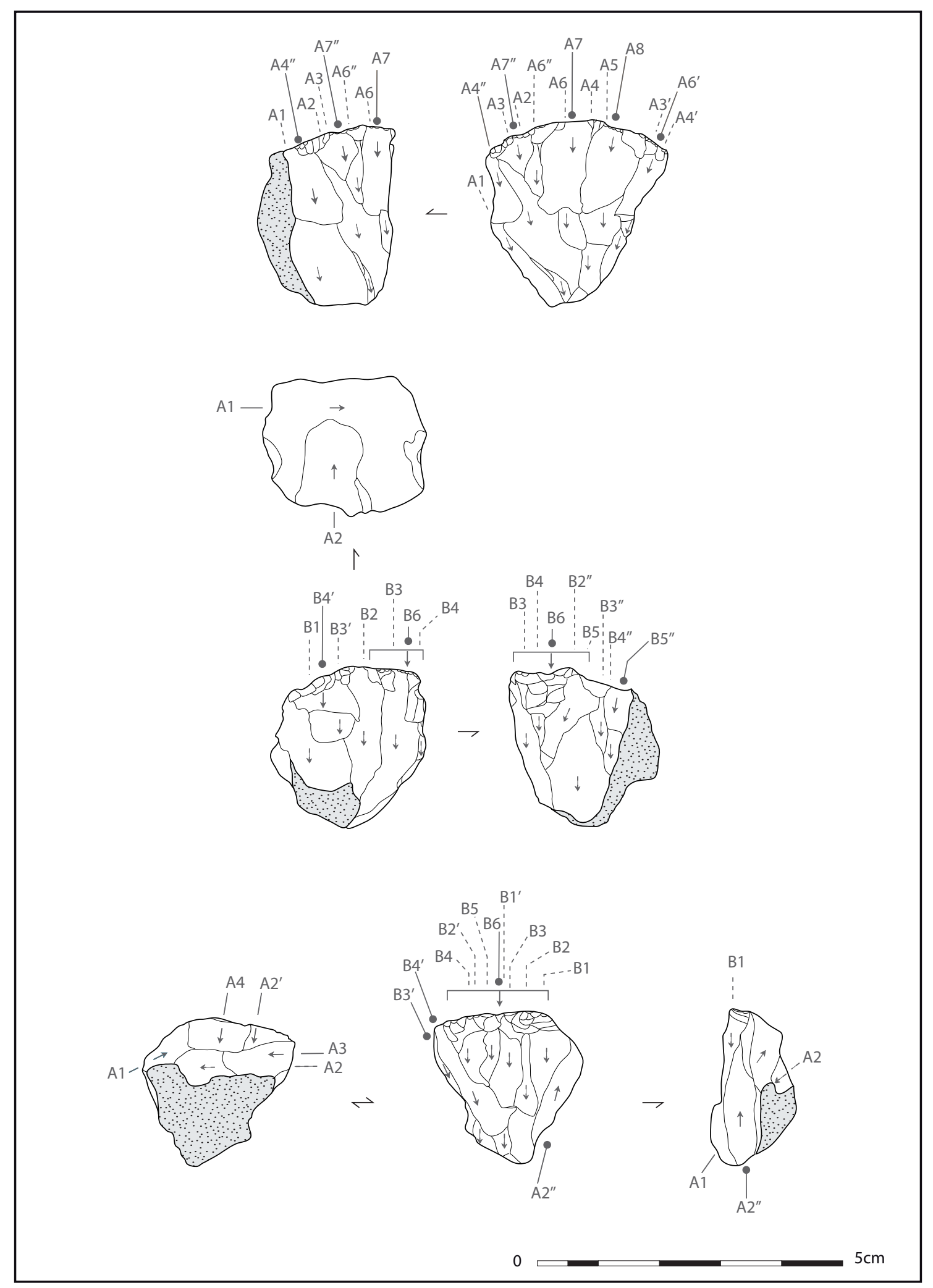

Figure 4 : Nucléus unipolaires semi-tournants.

Figure 4: Cores from the excavations. 


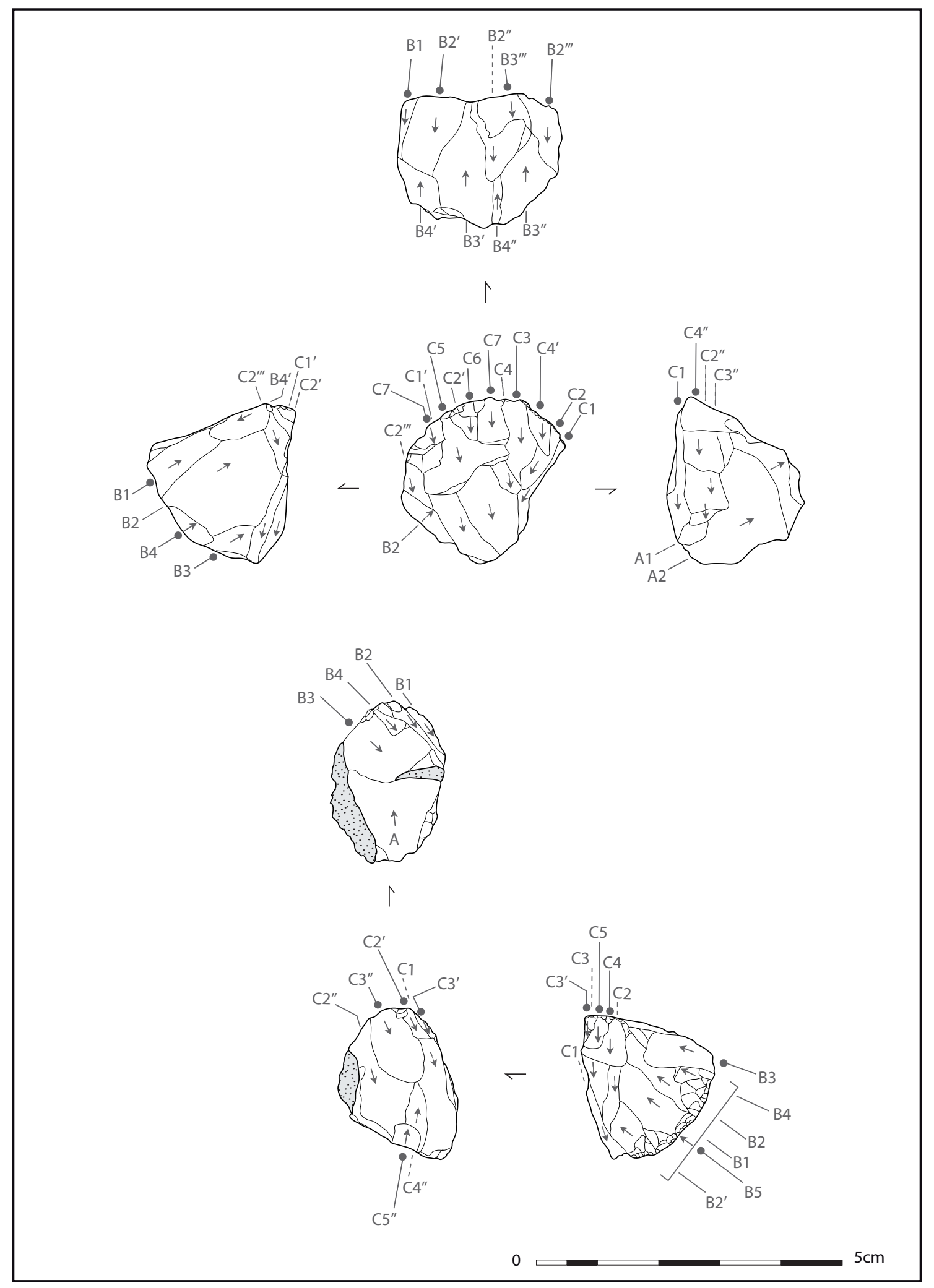

Figure 5 : Exploitation lamellaire à partir de deux plans de frappe.

Figure 5: Cores from the excavations 
fonctionnelle du site. En effet, la proportion d'outils se résumant à des enlèvements utilisés bruts est difficilement quantifiable. Aux Vingt-Deux Boisselées-1, le taux de vestiges à fil ébréché est non négligeable (tabl. 2) ${ }^{4}$, mais certains de ces esquillements sont peut-être dus à des processus taphonomiques relatifs à la nature du sédiment encaissant (sables, graviers et galets). En parallèle, il faut également considérer l'éventuelle utilisation de supports bruts dans des actions brèves et ne laissant que des traces visibles au microscope à fort grossissement. Les analyses fonctionnelles réalisées sur certains sites du début du Mésolithique dans le nord de la France et la Belgique mettent en effet en évidence l'importance de ce dernier type d'outils (Guéret, 2010). À la liste de ces arguments, d'autres paramètres mériteraient d'être ajoutés, dont le nombre inconnu d'armatures emmanchées sur chaque fût.

\section{Un outillage du fonds commun peu caractéristique}

Cette série illustre une fois de plus le fait que l'aspect sommaire de l'outillage du fonds commun constitue une caractéristique majeure des industries du début du Mésolithique, que l'on se focalise sur l'ouest de la France ou que l'on élargisse la fenêtre d'étude à l'Ouest européen. Dans le sud de la France, plusieurs publications insistent d'ailleurs sur l'importance du caractère expédient de l'outil chez les groupes mésolithiques (Philibert, 2002, p. 163; Guilbert et al., 2006). Pour en revenir au site qui nous intéresse dans le cas présent, la catégorie d'outils qui prédomine est celle des " enlèvements à retouches diverses ", pour ne pas dire celle des outils inclassables en l'absence d'analyses tracéologiques. Plus précisément, ces pièces correspondent à 54,8 \% des outils retouchés du fonds commun (tabl. 3 , fig. 6). Dans près de la moitié des cas, un éclat a été choisi comme support. On trouve parmi eux des enlèvements plus ou moins épais, et parfois à plages corticales, qui sont symptomatiques des phases de (ré)aménagement, depuis l'entame jusqu'au nettoyage de réfléchissements survenus lors de séquences de production lamellaire. D’autres supports, assez fins et de petite dimension, ne correspondent pas à des pièces techniques et ont certainement été extraits en amont des séquences lamellaires ( $c f$. supra). La retouche des différents éclats se situe en partie distale des supports ou sur leurs bords. En ce qui concerne les produits lamino-lamellaires qui ont été sommairement retouchés, les retouches se développent surtout sur un des bords, sans latéralisation préférentielle. Ces supports sont d'une régularité moyenne : si elle semble inférieure à celle affichée par les armatures et les microburins, elle surpasse globalement celle des produits

4. Sans pouvoir commenter la valeur de cette observation, on notera qu'au sein de cette catégorie les éclats fins, plus ou moins allongés, et d'un calibre de 1 à $4 \mathrm{~cm}^{2}$, sont bien représentés.

\begin{tabular}{|c|c|}
\hline Retouche diverse & 17 \\
\hline Coche isolée & 4 \\
\hline Troncature & 4 \\
\hline Grattoir & 2 \\
\hline Denticulé & 2 \\
\hline Burin & 1 \\
\hline Pièce à bord abattu & 1 \\
\hline TOTAL & $\mathbf{3 1}$ \\
\hline
\end{tabular}

Tableau 3 : Structuration en nombre de l'outillage commun. Tableau 3: Composition of the common tools.

lamino-lamellaires bruts (tabl. 4). L'épaisseur de ces outils [3,5-5,5 mm] et leur largeur, plus variable [7,5-17,5 mm], dépassent également le plus souvent les dimensions des supports réservés à la fabrication des microlithes.

Le reste du fonds commun est diversifié, avec des types d'outil constitués d'une ou de quelques pièces. Les deux grattoirs du corpus ont été réalisés par retouches directes semi-abruptes sur de grands éclats épais, l'un des supports résultant du réaménagement du nucléus en partie opposé au plan de frappe (fig. $6, n^{\circ} 6$ ). Le recyclage de cette pièce technique s'inscrit dans une logique de sélection des supports similaire à celle évoquée pour les outils précédemment décrits. Une lame épaisse semi-corticale sert de support à l'unique burin. Les quatre outils tronqués, latéralisés à droite, ont en revanche été fabriqués sur des lamelles et un éclat fin (fig. $6, \mathrm{n}^{\circ}$ 10). La délinéation de la troncature, l'angle qu'elle forme avec l'axe du support et l'inclinaison des retouches sont alors très variables. Les outils encochés sont également confectionnés sur des enlèvements fins. Parmi les quatre pièces à coche unique (fig. $6, \mathrm{n}^{\circ} 9$ et 11 ), une se démarque par son support, lamellaire, et le type de retouche, inverse. Les deux denticulés quant à eux ne possèdent respectivement que deux et trois coches successives (fig. 6, no 4).

\section{Un fonds microlithique dominé par des triangles isocèles}

Si l'on étudie l'ensemble des armatures provenant du sondage 1 , outre la prédominance du silex $(96,8 \%$ des armatures), on retient parmi les caractéristiques majeures $\mathrm{du}$ fonds microlithique le fort taux de triangles isocèles ( $51,4 \%$ des armatures ayant obtenu une attribution typologique (tabl. 5; fig. 7, $\mathrm{n}^{\circ} 1-16$ ). Si l'on commence par leurs attributs morphométriques, on peut apprécier une certaine constance de leur largeur : ses valeurs minimales et maximales, respectivement de 4 et $7 \mathrm{~mm}$, sont groupées autour de la moyenne, soit $5,6 \mathrm{~mm}$. Cette observation, récurrente dans l'ouest de la France et même parfois plus 


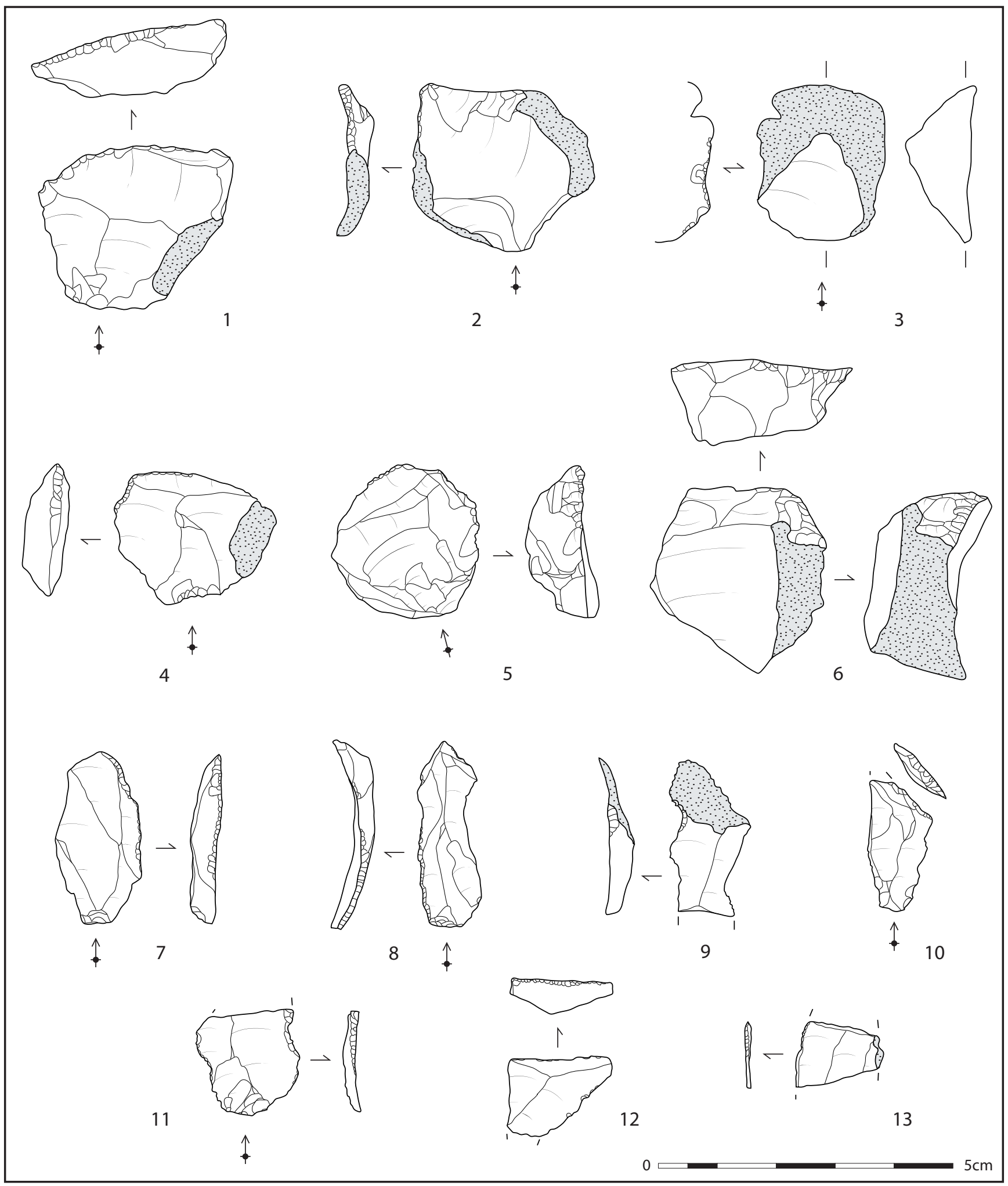

Figure 6 : Outils du fonds commun. 1-3,7, 8, 12, 13 : pièces à retouches diverses; 4 : éclat denticulé; 5,6 : grattoirs; 9,11 : supports lamino-lamellaires encochés; $10:$ lame tronquée.

Figure 6: Common tools. 1-3, 7, 8, 12, 13: retouched pieces; 4: denticulate flake; 5, 6: scrappers; 9, 11: notched pieces; 10: truncated blade. 


\begin{tabular}{|c|c|c|}
\hline Régularité & Lamelles brutes & Microburins \\
\hline-- & 32,6 & 7,3 \\
\hline- & 58,9 & 85,5 \\
\hline+ & 8,5 & 7,3 \\
\hline Total & 100 & 100 \\
\hline
\end{tabular}

Tableau 4 : Comparaison de la régularité des produits laminolamellaires bruts et des microburins.

Tableau 4: Comparison of the regularity of the blade(let)s and the microburins.

\begin{tabular}{|c|c|c|c|}
\hline Type & Sous-type & $\mathrm{N}$ & $\%$ \\
\hline \multirow{2}{*}{ Triangle } & scalène & 4 & 10,8 \\
\cline { 2 - 4 } & isocèle & 19 & 51,4 \\
\hline \multirow{2}{*}{ Pointe } & à base naturelle & 3 & 8,1 \\
\cline { 2 - 4 } & à base retouchée & 6 & 16,2 \\
\hline \multicolumn{2}{|c|}{ Armature segmentiforme } & 5 & 13,5 \\
\hline \multicolumn{2}{|c|}{ Sous total } & 37 & 100 \\
\hline \multicolumn{2}{|c|}{ Armature en cours de fabrication } & 1 & \\
\hline \multicolumn{2}{|c|}{ Fragment d'armature } & 24 & \\
\hline \multicolumn{2}{|c|}{ TOTAL } & 62 & \\
\hline
\end{tabular}

Tableau 5 : Composition générale du fonds microlithique.

Tableau 5: Composition of the arrowheads.

tranchée sur certains sites de ce secteur (Valdeyron, 1991; Michel, 2011, p. 367), doit être prise en considération pour s'interroger sur le mode d'emmanchement des armatures isocèles. L'exigence en termes de longueur est nettement moindre, ce qui explique la variation de l'allongement de ces armatures. L'indice allongement appartient à l'intervalle [1,6-2,9 ${ }^{5}$, mais en complément d'information il faut signaler que ce ratio se situe au dessus de 2,5 pour la majorité des triangles isocèles entiers; en d'autres termes, la préférence va aux modèles allongés plus qu'aux gabarits trapus. Quelques triangles isocèles se distinguent par leur caractère hypermicrolithique (fig. $7, \mathrm{n}^{\circ} 15-16$ ) qui rappelle des armatures découvertes à seulement quelques kilomètres, à l'autre extrémité du Boivre, à la Fillauderie (Saint-Père-en-Retz, Marchand et al., 1999). Pour être exhaustif d'un point de vue morphométrique, il convient également de préciser la faible épaisseur des supports choisis pour être transformés en triangles isocèles, cette dimension n'excédant pas sur ces microlithes 1 à $2 \mathrm{~mm}$ selon les cas. On peut enfin ajouter que les troncatures sont globalement rectilignes, que le troisième côté fait rarement l'objet d'un bordage et que, sur un

5. L'intervalle reste ouvert à son maximum, du fait de la fragmentation transverse ou oblique des microlithes qui affecte leur longueur totale. peu plus d'un cinquième des triangles isocèles, on observe encore un piquant-trièdre partiellement brut. Dans toutes les collections du Centre-Ouest, le piquant-trièdre a souvent été conservé (partiellement ou totalement) brut sur tous types d'armature. Pour les triangles, ce caractère constitue une spécificité régionale, le piquant-trièdre n'apparaissant qu'exceptionnellement sur les microlithes du Sauveterrien ancien (Valdeyron et al., 2008).

La catégorie des triangles est complétée par quatre armatures scalènes (fig. $7, \mathrm{n}^{\circ}$ 17-19). L'un d'entre eux tend fortement vers la symétrie et présente des caractéristiques semblables à celles exposées dans le paragraphe ci-dessus. Un autre, latéralisé à droite comme ses semblables, est plus allongé avec un indice d'allongement de 2,8. Le troisième côté est finement retouché sur ses trois quarts depuis la grande pointe, tandis que quelques millimètres avant la pointe la grande troncature s'interrompt au profit d'une micro-retouche. L'assimilation au sous-type "triangle de Montclus » ne se justifie pas pour autant, les caractères effilé et hypermicrolithique étant absents.

Un quart du fonds microlithique correspond à des pointes (fig. 7, n²3-32), les modèles à base retouchée étant les plus fréquents. Cette dernière est toujours orthogonale à l'axe de l'armature et de délinéation majoritairement concave, avec alors parfois un léger décentrage de l'arc vers le bord retouché. La mise en œuvre de retouches inverses, et non directes, est exceptionnelle. Le troisième bord est ponctuellement travaillé sur toute sa longueur ou seulement dans le tiers proche de la base. La largeur des pointes à base retouchée est constante, avec des valeurs comprises entre 6 et $8 \mathrm{~mm}$. En revanche, la variabilité est de mise aussi bien pour la latéralisation (gauche-droite) que pour l'implantation de la pointe (parties distale ou proximale). Ces deux remarques peuvent être réitérées pour les pointes à base naturelle, dont le nombre réduit ne permet certes pas de conclure sur ce point. Deux de ces microlithes, fracturés, ressemblent morphologiquement parlant à de réels triangles, si ce n'est qu'ils ne sont tronqués qu'une fois (fig. $7, n^{\circ} 24$ et 26). Ce type de pièces revient fréquemment, au travers de quelques exemples, dans les séries sub-contemporaines de l'ouest de la France. Faut-il les classer en type à part entière ou a-t-on affaire à des armatures en cours de fabrication? Aux Vingt-Deux Boisselées-1, les cassures, malheureusement non diagnostiques, ne permettent pas de nourrir le débat. Mais la dimension très réduite d'une des pointes irait à l'encontre de la seconde hypothèse. La dernière pointe à base naturelle possède également une particularité : c'est l'unique microlithe de cette série dont l'axe est orthogonal à celui du support (fig. $7, \mathrm{n}^{\circ} 25$ ). Le tailleur a profité de la cassure nette d'un fragment distal, en la perfectionnant sur une moitié avec des retouches directes abruptes, pour réaliser 


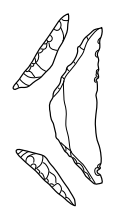

1

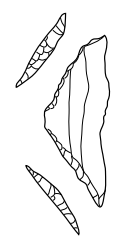

2

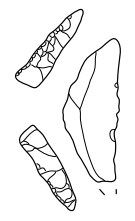

3

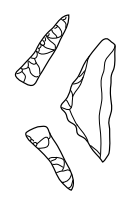

4

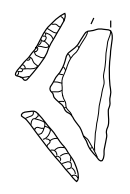

5

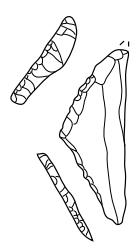

6

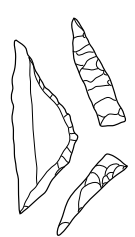

7

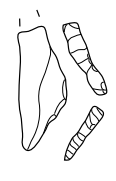

8

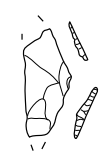

9

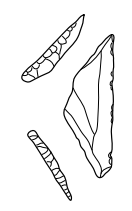

10

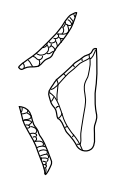

11

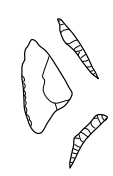

12

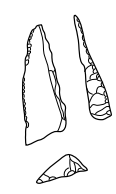

19<smiles>C1=CCCCC1</smiles>

26
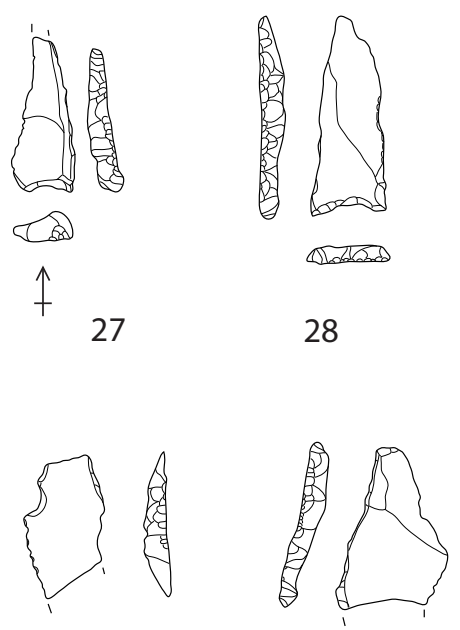

35

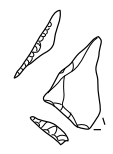

13

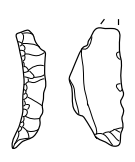

20

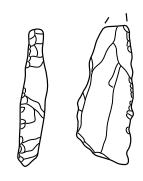

21

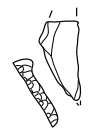

14<smiles>c1ccc(-c2ccccc2)cc1</smiles>

15
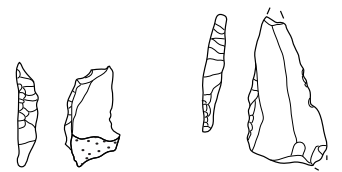

23

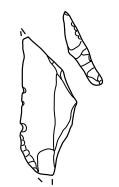

24

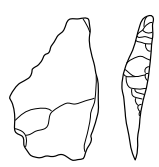

25

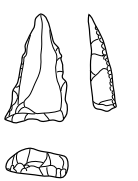

32

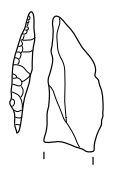

33

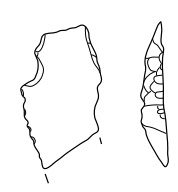

34
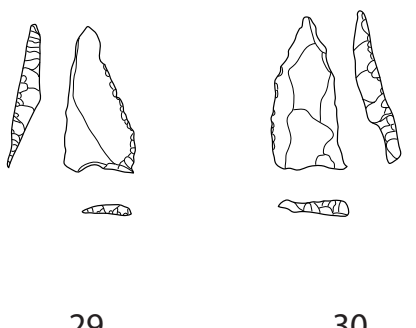

30

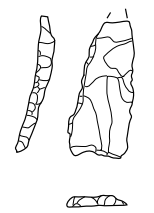

31

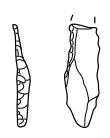

管

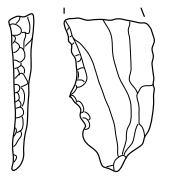

36

37

38

$5 \mathrm{~cm}$

Figure 7 : Armatures récoltées dans le sondage 1. 1-16: triangles isocèles; 17-19: triangles scalènes; 20-22: armatures segmentiformes; 23-26: pointes à base naturelle; 27-32 : pointes à base retouchée; 33-38: fragments d'armatures à troncature oblique ou bord abattu. Figure 7: Arrowheads from the excavations. 1-16: isosceles triangles. 17-19: scalene triangle. 20-22: segments. 23-26: points with natural base. 27-32: points with retouched base. 33-38: fragments. 
une pointe dont la base correspond au bord gauche de la lamelle-support.

Les armatures segmentiformes (fig. $7, \mathrm{n}^{\circ}$ 20-22), dont l'étroitesse rappelle celle des triangles isocèles, paraissent un peu moins régulières que les autres types de microlithes; c'est d'ailleurs dans cette classe qu'on trouve le seul projectile à plage corticale. Globalement, un axe de symétrie horizontal se dessine au milieu de la pièce (segments de cercle) mais une pièce se distingue par son asymétrie et peut être qualifiée de pointe segmentiforme. Pour information, aucun piquanttrièdre n'est alors apparent.

Cette dernière remarque nous amène à décrire brièvement quelques traits relatifs au procédé du microburin, renseigné par 61 pièces (microburins à proprement parler, lamelles à cassure dans ou au-dessus de la coche et lamelles encochées en partie mésiale). Le tailleur implante préférentiellement la coche à droite et de manière à supprimer le segment proximal (tabl. 6). Cette ablation facilite la confection des armatures débarrassées de la section la plus irrégulière et la plus épaisse du fait des bulbe et talon.

\section{Les Vingt-Deux Boisselées-1 : DE NOUVELLES PISTES D'ORGANISATION CHRONO-CULTURELLE CONCERNANT LES PREMIERS GROUPES MÉSOLITHIQUES DE L'OUEST DE LA FRANCE?}

En termes de choix techniques et d'objectifs de débitage, on note sur ce site que l'investissement ne porte pas tant sur la production des supports que sur leur retouche, ce qui semble globalement être le cas dans l'ouest de la France. La caractérisation technologique de la série des Vingt-Deux Boisselées-1 est également intéressante si l'on confronte les résultats de ce site localisé dans le sud du Massif armoricain à ceux des séries réalisées aux environs dans les bassins sédimentaires à partir de silex locaux. Les conclusions de cette comparaison ont déjà été présentées en détails à plusieurs reprises (Michel, 2011, p. 420-425; Michel, sous presse) et l'on peut juste retenir dans le cadre de cet article consacré à un site sud-ligérien que la spécificité géologique du

\begin{tabular}{|c|c|c|c|}
\cline { 2 - 4 } \multicolumn{1}{c|}{} & Proximal & Distal & TOTAL \\
\hline Gauche & 20,8 & 13,2 & 34 \\
\hline Droite & 49,1 & 16,9 & 66 \\
\hline TOTAL & 69,9 & 30,1 & 100 \\
\hline
\end{tabular}

Tableau 6 : Caractères des microburins exprimés en pourcentage. Tableau 6: Description of the microburins. sous-sol armoricain n’influe globalement ni sur les méthodes d'exploitation des matières siliceuses ni sur les objectifs de débitage.

Le second apport principal de cette opération concerne les armatures, qui pour le Mésolithique sont au cœur des caractérisations chrono-culturelles. La description générale du fonds microlithique tel qu'il apparaît au niveau du sondage diffère en effet nettement de celle qui peut être dépeinte si l'on se concentre sur la série récoltée en prospections aux Vingt-Deux Boisselées-1. Jusqu'alors, et d'après ces ramassages de surface, les pointes constituaient la principale composante du carquois de ce site $(60,5 \%$ hors pièces fragmentées ou en cours de façonnage). Dans le sondage 1, cette proportion s'applique au contraire aux triangles ${ }^{6}$. Comment appréhender cette distinction majeure en termes de détermination culturelle?

Tout d'abord, cette constatation nous amène à réitérer les conseils de prudence quant à l'emploi des statistiques pour les séries issues de prospection. Si l'on se recentre sur le site des Vingt-Deux Boisselées-1, on peut tout d'abord supposer, par extension, qu'une nette prédominance des triangles serait apparue si l'emprise de la fouille avait été de proportions égales à la zone ayant livré du matériel mésolithique en surface (fig. 8). Cependant, différents facteurs doivent être pris en compte. Nous ne sommes pas à l'abri par exemple, et pour ne citer que cet aspect, d'une répartition différentielle des indices archéologiques. Un second point doit être soulevé : la totalité des pointes du sondage proviennent des labours. Les triangles isocèles sont quant à eux répartis de manière équilibrée entre ces labours et le niveau sous-jacent. Faut-il y voir le spectre d'une stratigraphie? En d'autres termes, il serait tentant d'évoquer un niveau, escamoté par les travaux aratoires, renfermant pointes et triangles isocèles et un niveau antérieur au sein duquel seule la seconde catégorie d'armatures serait représentée (fig. 9). Le contexte sédimentaire ne permet pas de valider l'hypothèse de deux occupations successives caractérisées par des habitudes stylistiques différentes (une phase à triangles suivie d'une période où le carquois se diversifie). En revanche, il faut garder en mémoire cette possible stratigraphie résiduelle pour voir si elle se confirme sur des sites moins altérés. En effet, ce constat, qui mettrait en évidence une stabilité des modalités d'approvisionnement et de débitage alors que les microlithes évolueraient, ne serait pas sans conséquence sur les modèles chrono-culturels actuels.

6. Pour approcher de manière plus juste les proportions des types d'armatures, le raisonnement a également été mené de la façon suivante. L'emprise du sondage est de $4 \mathrm{~m}^{2}$, au sein de $2850 \mathrm{~m}^{2}$ livrant des vestiges mésolithiques en prospections. Pour chaque type d'armature, le calcul "nombre provenant du sondage 1 " + («nombre provenant des prospections » X 4 / 2 850) a été réalisé. Les triangles isocèles sont encore majoritaires. 


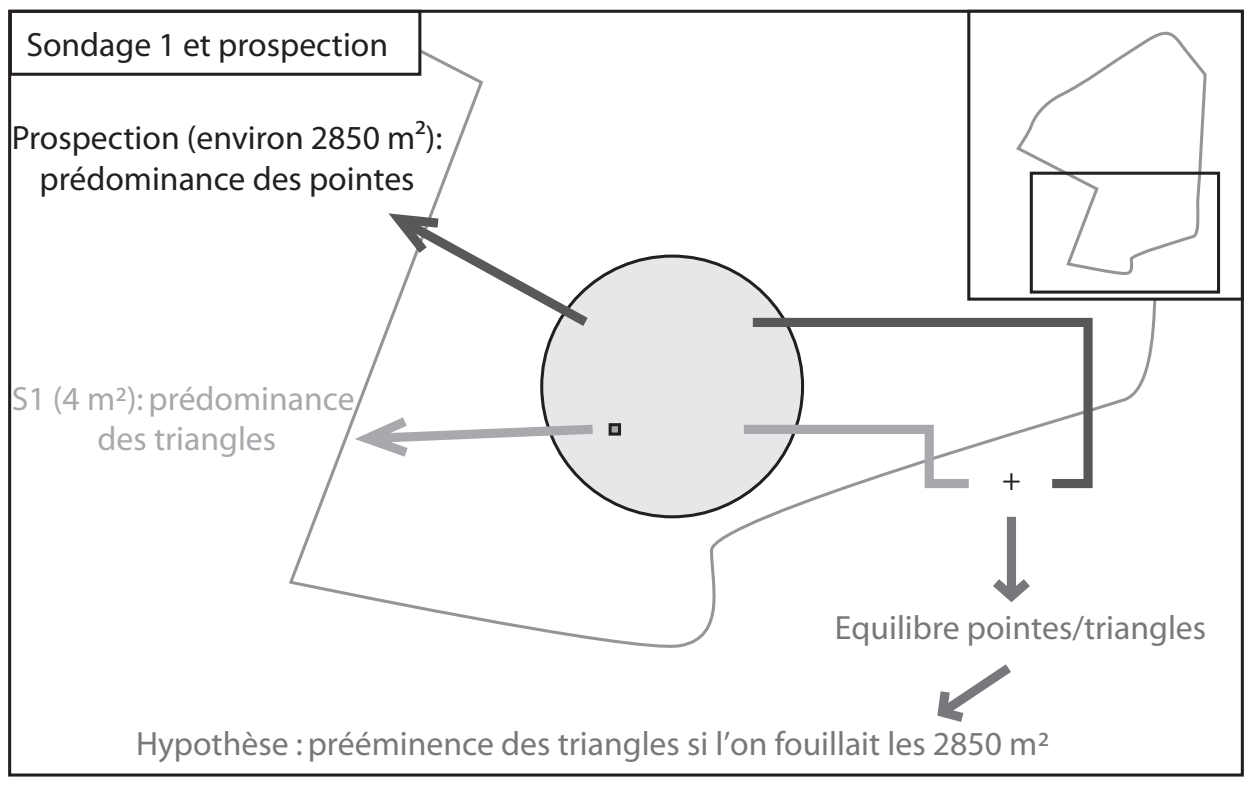

Figure 8 : Estimation des proportions des types d'armatures : comparaison entre les prospections et le sondage.

Figure 8: Estimation of the proportions of the types of arrowheads: comparison between the surveys and the excavation.
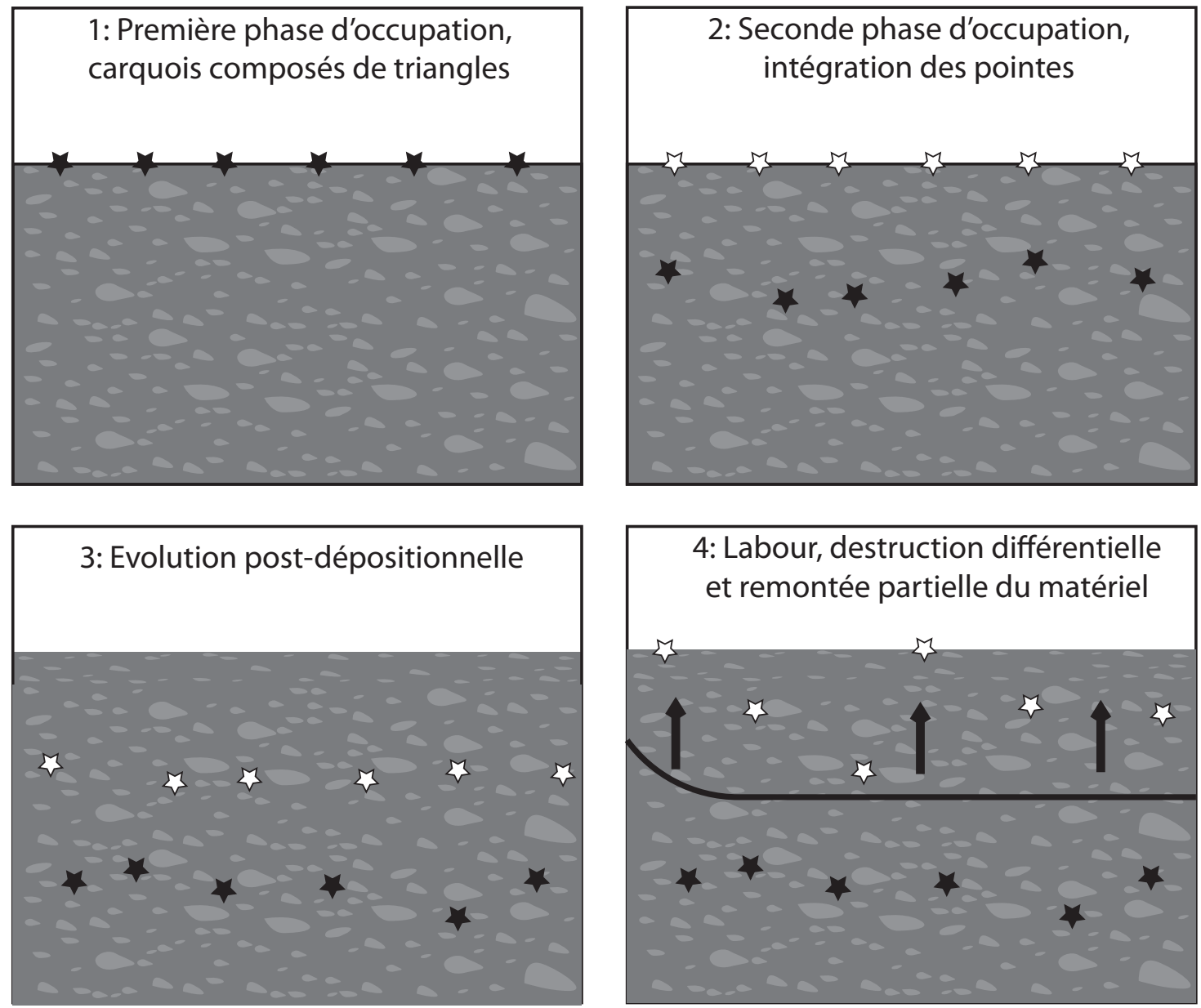

Figure 9: Schématisation d'une éventuelle diachronie des occupations.

Figure 9: Simplification of a possible diachrony of the occupations. 
Il convient à ce stade de la réflexion de souligner trois points. Tout d'abord, rappelons que dès 1984 O. Kayser proposait un classement successif des sites morbihannais de Kerjouanno (Arzon) et de Monterblanc (Guernéhué), en arguant de la hausse progressive des pointes parallèlement à la baisse des triangles.

Deuxièmement, il n'y a pas d'emploi d'une matière première donnée et différente pour chacun de ces types d'armatures, le silex blond étant largement prédominant dans l'ensemble du fonds microlithique. Nous sommes donc dans un cas de figure différent de la Majoire D (Gouraud, 1987), site régional associant des pointes à base retouchée majoritairement fabriquées en silex blond et d'autres types de microlithes généralement réalisés en grès-quartzites. On peut y suspecter 1) un usage différentiel des matières premières en fonction des classes d'armatures (cas de figure d'un seul et même ensemble), 2) une évolution des systèmes d'approvisionnement en matériaux siliceux en parallèle d'une modification des normes typologiques (cas de figure d'une diachronie), ou 3) le passage sur le site de deux groupes culturels différents comme cela a pu être proposé dans l'est de la France à partir de classements typologiques (Thévenin, 1998); mais le contexte de découverte, c'est-à-dire des ramassages de surface, ne permet pas de privilégier une de ces hypothèses.

Enfin, aux Vingt-Deux Boisselées-1 les pointes à base naturelle peuvent être mieux caractérisées grâce aux prospections qui en ont livré 17 exemplaires. L'impression d'opportunisme née de l'étude du matériel issu du sondage disparait au profit d'un modèle de pointes plus récurrent. Il se résume à la combinaison d'une latéralisation préférentiellement senestre, d'un apex implanté en partie distale, d'une délinéation rectiligne ou concave de la troncature et d'un bord opposé non aménagé. En revanche, seules deux de ces armatures correspondent à la définition des pointes de Chaville, le piquant trièdre n'étant absolument pas retouché.

En guise de conclusion, on soulignera qu'avec l'exemple des Vingt-Deux Boisselées-1 on voit à quel point des sondages d'emprise réduite, réalisés dans des contextes géomorphologiques assez peu favorables à l'enregistrement des données, peuvent tout de même livrer d'importants résultats permettant de compléter notre vision des premiers chasseurs-collecteurs mésolithiques de l'actuelle façade atlantique française.

\section{Bibliographie}

Arrivé, L., Godard, G., Dugast, C., Gouraud, G. et Jauneau, J.-M., (en préparation) - Approche géologique des différents matériaux utilisés en Vendée par les groupes préhistoriques.

Barton, R. N. E., 1987 - Vertical distribution of artefacts and some post-depositional factors affecting site formation, in Rowley-Conwy, P., Zvelebil, M. et Blankholm, H. P. (dir.), Mesolithic Northwest Europe: recent trend. University of Sheffield, Department of Archaeology and Prehistory, p. 55-62.

Crombe, P., 1993 - Tree-fall features on final Palaeolithic and Mesolithic sites situated on sandy solls : how to deal with it, Helinium, 28, p. 50-66.

Gallais, J.-Y., Damblon, F., Richard, J., Thiébault, S. et Visset, L., 1985 - Le site à microlithes de l'Organais en Sainte-Reine de Bretagne (Loire-Atlantique), Revue archéologique de l'Ouest, 2, p. 23-45.

Gouraud, G., 1987 - Le Mésolithique des Majoires à Montbert (Loire-Atlantique). La station D, Groupe vendéen d'Études préhistoriques, 18, p. 14-41.

Gouraud, G., 1990 - Rapport sur le sondage n 90-17, site 44-223-01 AP, Les Garennes, manuscrit, 7 p.

Gouraud, G., Dugast, C., Jauneau, J.-M. et Ricoulleau, P.-I., 1990 - Trois nouveaux sites à microlithes à Beaufou (Vendée), Groupe vendéen d'Études préhistoriques, 23, p. 6-30.

Gueret, C., 2010 - Identité et variabilité de l'outillage lithique du Premier Mésolithique en Belgique et dans le Nord de la France : les apports de l'approche fonctionnelle, in Valentin, B., Ducroce, T., Fagnart, J.-P., Séara, F. et Verjux, C. (dir.), Palethnographie du Mésolithique : recherches sur les habitats de plein air dans la moitié septentrionale de la France et ses marges, communication orale, 26-27 novembre 2010, Paris.

Guilbert, R., Philibert, S. et Peresani, M. 2006 - L'outil expédient : une partie intégrante de la production mésolithique, in Astruc L., Bon F., Léa V., Milcent P.-Y. et Philibert S. (dir.), Normes techniques et pratiques sociales, de la simplicité des outillages pré-et protohistoriques, $\mathrm{XXVI}^{\mathrm{e}}$ rencontres internationales d'Archéologie et d'Histoire d'Antibes, Antibes, Éditions APDCA, p. 161-171.

Guyodo, J.-N. et Marchand, G., 2005 - La percussion bipolaire sur enclume dans l'ouest de la France de la fin du Paléolithique au Chalcolithique : une lecture économique et sociale, Bulletin de la Société préhistorique de France, t. 102, 3, p. 539-549.

LE Goff, G., 2002 - Une étape de l'histoire des techniques : l'étude technologique du locus 1 du site mésolithique ancien des VingtDeux-Boisselées, Saint-Père-en-Retz (Loire-Atlantique), Mémoire de maîtrise, Lorient, Université de Bretagne Sud.

LE Goff, G., 2003 - Le site mésolithique de l'Organais, SainteReine-de-Bretagne (LoireAtlantique) : étude technologique, Mémoire de DEA, Rennes, Université Rennes 2. 
Marchand, G., 1997 - La néolithisation de l'Ouest de la France : caractérisation des industries lithiques, Thèse de doctorat, université Paris I.

Marchand, G., Tessier, M. et Bernard, J., 1999 - Les occupations mésolithiques et neolithiques de la Fillauderie (SaintPère-en-Retz, Loire-Atlantique) et la préhistoire récente de la basse vallée du Boivre, Revue archéologique de l'Ouest, 16, p. 39-65.

Michel, S., avec la collaboration de David Aoustin, Laurent Charrieau, Chantal Leroyer et Jean-Charles Oillic, 2009 Les Vingt-Deux Boisselées (Saint-Père-en-Retz) : une occupation du Mésolithique à pointes et triangles en pays de Retz, rapport d'évaluation 2009, Service régional de l'Archéologie Pays-dela-Loire, $41 \mathrm{p}$.

MiCHEL, S., 2011 - Les premiers groupes mésolithiques de la France atlantique : enquête sur l'industrie lithique, Thèse de doctorat, université Rennes 1.

Michel, S., (sous presse) - Silex, grès-quartzite et phtanite au Mésolithique ancien entre Loire et Charente : comportements techniques et gestion économique, in Marchand, G. et Querré, G. (dir.) Roches et sociétés de la Préhistoire en entre Massifs cristallins et Bassins sédimentaires, 28-30 avril 2010.

Pelegrin, J., 2000 - Les techniques de débitage laminaire au Tardiglaciaire : critères de diagnose et quelques réflexions, In Valentin B., Bodu P. et Christensen M. (dir.), L’Europe centrale et septentrionale au Tardiglaciaire, Actes de la table ronde internationale de Nemours, 14-16 mai 1997, APRAIF, Mémoire du musée de Préhistoire d'Île-de-France, t. 7, Nemours, p. 73-86.

Philibert, S., 2002 - Les derniers "sauvages": territoires économiques et systèmes technofonctionnels mésolithiques, British Archaeological Reports, International Series 1069, Oxford, Hadrian Books, 191 p.

Ters, M., Marchand, J., Châteauneuf, J.-J., Visset, L., Biteau, F., Отtmann, F., Limmaset, O., Talbo, H., Guigues, J. et Bambier, A., 1978 - Paimbouf, estuaire de la Loire et Paysde-Retz. Notice explicative de la carte géologique de la France à 1/50000. Bureau de Recherches géologiques et minières, Orléans, 59 p.
Ters, M., Mounès, J., Ollivière-Pierre, M.-F., Féraud, J., Gautier, M., Cailleux, A., Limasset, O., Andreieff, P. et Margerel, J.-P., 1979 - Machecoul. Notice explicative de la carte géologique de la France 1/50000. Bureau de Recherche Géologiques et Minières, Orléans, 37 p.

Tessier, M., 1998 - Saint-Père-en-Retz (Loire-Atlantique). Les 22 Boisselées, un site mésolithique ancien, Société d'Histoire et d'Archéologie de la plaine de l'Ain, 16, p. 64-71.

ThéVEnin, A., 1998 - Le Mésolithique du centre-est de la France : chronologie, peuplement, processus évolutifs, Revue archéologique de l'Est, 49, p. 87-133.

VAldeYron, N. 1991 - Typologie statistique des armatures triangulaires sauveterriennes : Fontfaurès et le contexte de la France méridionale, in Barbaza M., Valdeyron N., André, J., Briois, F., Martin, H., Philibert, S., Allios, D. et Lignon, E., Fontfaurès en Quercy, Paris, EHESS, coll. « Archives d'Écologie Préhistorique; 11 », p. 171-227.

Valdeyron, N., Bosc-Zanardo, B. et Briand, T., 2008 Évolution des armatures de pierre et dynamiques culturelles durant le Mésolithique dans le Sud-Ouest de la France : l'exemple du Haut Quercy (Lot, France), in Pétillon, J.-M., Dias-Meirinho, M.-H., Cattelain, P., Honegger, M., Normand, C., et Valdeyron, N. (coord.), Recherches sur les armatures de projectiles du Paléolithique supérieur au Néolithique (actes du colloque C83, XV congrès de l'UISPP, Lisbonne, 4-9 septembre 2006), P@lethnologie, 1, p. 278-295.

VermeErSCH, P.-M., 1976 - La position lithostratigraphique et chronostratigraphique des industries épipaléolithiques et mésolithiques en Basse Belgique. Congrès préhistorique de France, $\mathrm{XX}^{\mathrm{e}}$ session 1974, Provence, Paris, Société Préhistorique Française, p. 616-621.

Vermeersch, P.-M., 1995 - Processus post-dépositionnels sur des sites épipaléolithiques et mésolithiques en régions sableuses de l'Europe de l'ouest, in Thévenin A. et Bintz P. (dir.) L'Europe des derniers chasseurs, peuplement et paléoenvironnement de l'Epipaléolithique et du Mésolithique, actes du $5^{\mathrm{e}}$ colloque international UISPP, commission 12, Grenoble, 18-23 septembre 1995, Documents préhistoriques 12, Paris, Comité des travaux historiques et scientifiques, p. 159-166. 